\title{
Evolutionary multi-objective scheduling for anti-spam filtering throughput optimization
}

\author{
David Ruano-Ordás ${ }^{1,2}$,Vitor Basto-Fernandes ${ }^{3,4}$, Iryna Yevseyeva ${ }^{5}$, José Ramón \\ Méndez ${ }^{1,2}$ \\ ${ }^{1}$ Department of Computer Science, University of Vigo, ESEI, Campus As Lagoas, 32004 \\ Ourense, Spain \\ ${ }^{2}$ Centro de Investigaciones Biomédicas (Centro Singular de Investigación de Galicia), Campus \\ Universitario Lagoas-Marcosende, 36310 Vigo, Spain \\ ${ }^{3}$ Instituto Universitário de Lisboa (ISCTE-IUL), University Institute of Lisbon, ISTAR-IUL, \\ Av. das Forças Armadas, 1649-026 Lisboa, Portugal \\ ${ }^{4}$ School of Technology and Management, Computer Science and Communications Research \\ Centre, Polytechnic Institute of Leiria, 2411-901 Leiria, Portugal \\ ${ }^{5}$ Cyber Technology Institute, School of Computer Science and Informatics, Faculty of \\ Technology, De Montfort University, LE1 9BH Leicester, United Kingdom \\ drordas@uvigo.es, vitor.basto.fernandes@iscte.pt, \\ iryna.yevseyeva@dmu.ac.uk, moncho.mendez@uvigo.es
}

\begin{abstract}
This paper presents an evolutionary multi-objective optimization problem formulation for the anti-spam filtering problem, addressing both the classification quality criteria (False Positive and False Negative error rates) and email messages classification time (minimization). This approach is compared to single objective problem formulations found in the literature, and its advantages for decision support and flexible/adaptive anti-spam filtering configuration is demonstrated. A study is performed using the Wirebrush4SPAM framework anti-spam filtering and the SpamAssassin email dataset. The NSGA-II evolutionary multi-objective optimization algorithm was applied for the purpose of validating and demonstrating the adoption of this novel approach to the anti-spam filtering optimization problem, formulated from the multi-objective optimization perspective. The results obtained from the experiments demonstrated that this optimization strategy allows the decision maker (anti-spam filtering system administrator) to select among a set of optimal and flexible filter configuration alternatives with respect to classification quality and classification efficiency.
\end{abstract}

Keywords: Rule-based anti-spam systems, scheduling, multi-objective optimization.

adfa, p. 1, 2011.

(c) Springer-Verlag Berlin Heidelberg 2011 


\section{Introduction}

SPAM embraces the wide amount of unwanted communications delivered through Internet annoying most users. Some statistics about spam e-mail [1, 2] revealed the real dimension of this trouble. As shown in the work of Statista [1], in the period 2014-2016 the percentage of spam deliveries has kept beyond 52.5\%. Moreover, Digital Marketing Ramblings (DMR) stated that workers receive an average of 121 messages per day whereupon more than 63 are spam.

During the last years SpamAssassin filtering framework [3] become very popular in the Internet community to fight against spam. It implements a rule-based filtering method designed to combine multiple techniques. Rule-based anti-spam filtering allows the administrator to define ad hoc rules containing logical expression (used as a trigger) together with an associated score (importance in the classification process). Every time an e-mail is received for evaluation, all scores of rules matching the target message are summed. If the summation reaches a value over a predefined threshold (known as required_score) the incoming message is classified as spam by the filter. Otherwise, it is labelled as legitimate (also known as ham).

In this context, and given the extensive utilization and increasing significance of rule-based filtering frameworks for the anti-spam domain, several studies have addressed the optimization of parameters (rule scores and scheduling plan) to improve their accuracy [4, 5, 6, 7] and classification throughputois] $[8,9,10]$. However, previous works on throughput optimization are based on simple heuristics without taking into account its relation to accuracy. Keeping in mind this background, this work includes a preliminary study of addressing both optimizations in an unified form and using a Multi-objective Evolutionary Algorithm (MOEA).

Due to the high amount of rules being executed by the anti-spam filter (from hundreds to thousands) for the classification of each individual email message, and the difficulty of running a real anti-spam filtering system in the context of optimization experiments, we decided to adopt in this study a recently released configuration simulator [11]. In our formulation, rules to be executed are considered the tasks to perform and computational resources (CPU, RAM and IO) the assets required for rules execution against the email messages to be classified. Moreover, we consider the scores of the rules in the optimization process, because a score of 0 indicates that the rule could be dropped, contributing to reduce the time required to classify a message. Classification time (time required to execute all rules on an email message), false positive (FP) and false negative (FN) errors are the criteria to optimize (minimize) in our study.

The multi-objective problem formulation is characterized by several conflicting goals, for which not simply a single optimum solution, but a set of potential optimal solutions are obtained. These solutions represent the best trade-offs between the objectives and are given to the decision maker to decide which solution matches the trade-off of his/her interest. In multi-objective optimization an optimal solution can only be improved in one objective at the expense of loss in other(s). It is in the best interest of the decision maker that the multi-objective optimization approach provides 
him/her the ability to select among several optimal solutions with a diversity of tradeoffs among the objectives.

In order to evaluate the suitability of the multi-objective approach to the anti-spam filtering throughput optimization problem, and to compare the performance of this type of metaheuristics with best known results in the anti-spam filtering throughput optimization problem, we used NSGA-II [12]. NSGA-II is one of the most popular Evolutionary Multi-Objective Optimization Algorithms (EMOA), has been cited in 9341 papers [13], is among the most cited papers in IEEE Transactions on Evolutionary Computation, has been used in a large variety of multi-optimization problem types, is one of the representative algorithms of EMOA type, and is therefore most suited to be adopted in our study, at this stage of our research hypothesis.

The rest of the paper is structured as follows: Section 2 studies current background on filter accuracy and speed optimization, Sections 3 and 4 present the formulation of our proposal and the experimental results, Section 5 provides a detailed discussion of results and finally, Section 6 outlines the main outcomes and future work.

\section{State of the Art}

As mentioned above, previous work on optimization of accuracy and throughput have not been addressed in a joint form. This section includes a description of previous work addressing throughput (subsection 2.1) and accuracy (section 2.2) optimization.

\subsection{Throughput optimization}

Despite general advices provided by SpamAssassin team [14], the introduction of Wirebrush4SPAM [15] provided some practical advances to really improve the throughput of rule-based filters. Among all proposals, we highlight Smart Filter Evaluation (SFE), parallel rule execution and preventing the execution of rules having a score of 0 . SFE provides a lazy evaluation scheme that avoids the evaluation of some rules in specific circumstances. The execution of rules in parallel allows to efficiently combine the execution of rules with high computational requirements (CPU usage) together with rules that imply an I/O operation.

To take more advantage of the above mentioned advances (i.e. achieve the SFE conditions to stop a filter evaluation early and achieve an adequate parallelization of rules), some scheduling heuristics have been introduced $[8,9,10]$.

Concretely, [8] introduces five scheduling mechanisms (GAV, GDV, PFS, NFS and PSS) to handle the arrangement of the filtering rules according to different sorting criteria designed to take advantage of SFE technique together with the multiprocessing capabilities of current CPUs. The experimental results carried out over several filter configurations demonstrated the suitability of adjusting the rule execution order to save computational resources and achieve a fast filtering ( time savings between $13 \%$ and $26 \%$ ).

Nevertheless the growth trend of the e-mail deliveries in the last years forces to continuously increase the spam filtering throughput. To this end, authors in [10] proposed two scheduling strategies (RBM and CEM) for optimizing the time needed 
to classify new incoming e-mails through an intelligent management of computational resources. To accomplish this task, both strategies use the information about CPU usage and $\mathrm{I} / \mathrm{O}$ delay of each rule to achieve an adequate execution balance by combining rules that execute $\mathrm{I} / \mathrm{O}$ operations together with those using intensively CPU resources (perform complex computations). Additionally, in order to increase even more the performance of the previous scheduling strategies, authors in [10] proposed a novel heuristic ensemble method (also called MHE) able to hybridise two individual heuristics to make the most of them. The main difference with previous approaches is that MHE is a heuristic combinatorial scheme instead of a simple heuristic. MHE combines a main heuristic $(h)$ with an auxiliary one $(h$ ') for sorting rules in those situations in which $h$ is not able to break the tie between two rules. Using the MHE criteria, rule $r_{1}$ will be executed before any other rule $r_{2}$ when Equation 1 is true. Otherwise $r_{2}$ will be executed before $r_{1}$.

$$
h\left(r_{1}\right)>h\left(r_{2}\right) \vee\left(\left(h\left(r_{1}\right)=h\left(r_{2}\right) \wedge h^{\prime}\left(r_{1}\right)>h^{\prime}\left(r_{2}\right)\right)\right.
$$

where $h$ and $h$ ' represents the main and auxiliary heuristics respectively.

Due to the definition of the MHE criteria, the user must select both the main heuristic, as well as another one as auxiliary measure.

The results achieved by the RBM and CEM schemes did not improve the performance achieved in [8] due to the high time variability of $\mathrm{I} / \mathrm{O}$ operations. However, the execution of MHE combined with GDV and RBM allows to filter emails up to $10 \%$ faster than using any other alternative. This fact enable us to conclude that resource consumption heuristics are the most suitable alternative when used as secondary heuristic.

\subsection{Filter accuracy optimization}

Filter accuracy optimization was early addressed by the SpamAssassin. Evolutionary Algorithms (EA) were used in versions up to 3.0 and after 3.3.0 whilst the rest of the versions used a simple neural network (Perceptron) [16]. EA emerged over other optimization techniques mainly due to their ability to search in large continuous and combinatorial spaces and find approximate (near) optimal solutions [17].

However, due to some limitations of SpamAssassin optimization methods, some interesting proposals emerged from scientific context. Grindstone4SPAM [4] is a set of tools designed to aid in the configuration and deployment of SpamAssassin filters. It includes a rules score optimization tool based on the usage of a Single Objective Evolutionary Algorithm (SOEA). The main limitation of SOEAs is the need of apriori establishing the criterion for a good solution. In this sense, in some environments the existence of FP errors could be inadmissible, other may prefer reduce the overall number of errors $(\mathrm{FP}+\mathrm{FN})$ or adequate balance between them.

With regard to this issue, EA are now widely accepted methods to search for approximations of optimal sets, in particular for dealing with multi-objective problems characterized by several conflicting goals, for which not simply a single optimum solution, but a set of optimal solutions need to be obtained. These solutions represent the trade-offs between the existing objectives, being optimal in the sense of 
so called Pareto dominance. A Pareto optimal solution can only be improved in one objective at the expense of loss in other(s). Since the population of solutions is used in parallel to solve these problems, the search is directed to not a single optimum but towards multiple Pareto optimal solutions, which is the case of EMOA.

A multi-objective optimization problem can be generically formulated as having $m$ objective functions, $f=\left(f_{1}, f_{2}, \ldots, f_{m}\right)$, which are simultaneously optimized (minimized or maximized) so that $f_{k}, k \in\{1, \ldots, m\}$ are real-valued functions evaluated in the multi-objective space. Additionally, constraints (equalities or inequalities) can be considered to impose restrictions on the decision variables. Decision variables are in this context the inputs given to the objective functions. Multi-objective optimization algorithms provide approximations to the set of Pareto optimal solutions that correspond to the set of non-dominated solutions found, based on so called Pareto dominance relation. A Pareto solution dominates any other $y$ alternative $\left(y, y^{\prime} \in R^{m}\right)$ if $y$ is better on at least one objective, and is not worse in the remaining objectives. The selection of a single solution among the set of Pareto optimal solutions is done by the decision maker(s) among the final Pareto optimal solutions given by the EMOA.

Taking into consideration the benefits of EMOAs, several works have analyzed in detail their usage to optimize scores with different problem formulations (scenarios). In [5], the authors provide a detailed comparison between the usage of NSGA-II and SPEA2 to reduce FP and FN errors (2 objectives). This work also compares these results with the ones achieved by using Grindstone4SPAM. Moreover, in [6] multiobjective optimization of classifiers by means of 3D convex-hull-based evolutionary algorithms was applied to different optimization problems, including spam filtering, revealing that several types of EMOA can achieve high quality solution sets when applied to the anti-spam filtering domain. Finally, in [7] authors introduce two different problem formulations with 3 optimization objectives. In the first formulation, rules can be activated or deactivated and the optimization objectives were the minimisation of the number of evaluated rules, FP and FN errors. The second formulation considers a three-way classification scheme and the optimization objectives were the reduction of unclassified samples, FP and FN errors.

Taking into consideration the relevance of EMOAs in literature, this work takes advantage of this type of algorithms to provide an efficient solution to optimize classification throughput and accuracy. Our proposal is introduced in the next section.

\section{Problem formulation and proposal}

In order to study the advantages of applying the EMOA approaches for the multiobjective anti-spam filtering scheduling problem formulation, we defined a scenario where classification quality and classification efficiency are optimized.

In this study, the anti-spam filtering problem is formulated as a multi-objective optimization problem characterized by real-valued objective functions $f(y)$ with values representing the number of false negative errors (FN, spam messages classified as legitimate), number of false positives errors (FP, legitimate messages classified as spam), and classification time (time to evaluate all the rules belonging to the filter). 
Note that these objectives are in conflict, since minimizing the number of FP can be done at the expense of increasing the number of FN and vice versa, and reducing the classification time can be achieved by ignoring irrelevant rules of the filter (rules having a score of 0 ) at the expenses of less accurate messages classification.

Minimization is assumed for all the objectives, which are evaluated with individuals collected from decision space as a two-vector decision variables space $y_{1}$, $y_{2}, \ldots, y_{n}$ where $i \in\{1, \ldots, n\}$ and $z_{1}, z_{2}, . ., z_{n}$ where $i \in\{1, \ldots, n\}$. These two vectors are represented by an array of decision variables, $y$ of length $n$ (the total number of filtering rules), where each variable $y_{i}$ corresponds to the score of one rule, and each variable $z_{i}$ indicates the order in which the rule must be executed (lower values meaning the rule is executed earlier). The individuals that are part of the initial population are randomly generated with scores in the $[-5,5]$ real variable range and execution orders a permutation variable in the range $[0, n]$ setting an execution ordering (scheduling plan) for rules execution. Additionally, new individuals are further generated by using the crossover and mutation operators in the same range. The rules scores range $[-5,5]$ and required_score threshold (5) was adopted in our study, because it is a common configuration for the operation of reference rule-based anti-spam filter systems such as SpamAssassin [3] and Wirebrush4SPAM. [15].

\section{$4 \quad$ Experimental study}

To carry out the optimization process we design a spam filter comprised by a total of 178 filtering rules ( 9 belonging to Naïve Bayes techniques and 169 corresponding to Regular Expressions). We decided to avoid rules belonging to network tests (like SPF or RBL/RWL techniques) due to variable latency of the network.

Table 1. Description of popular corpora on spam-filtering

\begin{tabular}{|l|c|c|c|c|}
\hline \multicolumn{1}{|c|}{ Corpus } & Duplicates & Size & \%Spam & \%Ham \\
\hline SpamAssassin [18] & $\mathbf{x}$ & 9332 & $74.5 \%$ & $25.5 \%$ \\
\hline CSDMC2010 [19] & $\mathbf{x}$ & 4327 & $68.1 \%$ & $31.9 \%$ \\
\hline 2055TRECSpam [20] & $\checkmark$ & 92189 & $57 \%$ & $43 \%$ \\
\hline 2006TRECSpam [20] & $\checkmark$ & 37822 & $65 \%$ & $35 \%$ \\
\hline 2007TRECSpam [20] & $\checkmark$ & 75419 & $66.5 \%$ & $33.5 \%$ \\
\hline Bruce Guenter [21] & $\checkmark$ & $>1000000$ & $100 \%$ & $0 \%$ \\
\hline Enron [4] & $\mathbf{x}$ & 517401 & $0 \%$ & $100 \%$ \\
\hline
\end{tabular}


Regarding to the dataset, we have studied a group of well-known corpora available online and distributed following the RFC 2822 [17] format. This RFC was proposed in 2001 and defines the syntax for representing e-mail messages. The raw text representation of messages following this syntax allows to easily parse and extract the required information. Table 1 shows a compilation of corpus following the RFC 2822 format together with relevant information about the size, percentages of spam and ham messages and the existence of duplicate e-mails.

As shown in Table 1, the first five corpora are constituted by both spam and legitimate emails while the remaining are single-class datasets (spam or ham) with a huge volume of messages. In order to save computation resources during experimentation without compromising the performance, medium-size datasets are highly recommendable. Taking into account these issues and also guaranteeing the independence of the dataset, the well-known SpamAssassin corpus [19] has been widely used in previous successful research works $[4,5,11,14]$. In order to ensure the reproducibility of results, we will also use this corpus for the experimental study.

The inclusion of an intelligent classification technique such as Naïve Bayes requires the use of a training corpus. To accomplish this, we divided SpamAssassin corpus into train/test groups following the same spam/ham ratio. Table 2 shows the final distribution used to accomplish our experimental protocol.

Table 2. Final corpus distribution

\begin{tabular}{|r|c|c|}
\hline & Spam & Ham \\
\hline Training set & 5215 & 1781 \\
\hline Test set & 1737 & 599 \\
\hline$\Sigma$ & 6952 & 2380 \\
\hline
\end{tabular}

As can be seen from Table 2, 75\% of the dataset is used for training Naïve Bayes while the remaining $25 \%$ is used for testing purposes.

Additionally, to perform this study, we used RuleSIM, a recent and complete toolkit for simulating the operation of rule-based anti-spam filters [23].

Being one of the reference and general purpose EMOA, one of the most referred in the computer science area in general and in the EMO area in particular, NSGA-II [12] was the algorithm adopted in our experiments. The main purpose of selecting this algorithm is, at this stage, to demonstrate and validate the application of EMOA approaches to the anti-spam classification efficiency domain.

The experiments were performed with jMetal 4.5.2 [24], an optimization framework for the development of multi-objective metaheuristics in Java. NSGA-II population size was set to 100 individuals and a maximum number of 10000 function evaluations was defined. Additionally, the SBX crossover and polynomial mutation operators were used to manipulate the real data decision variables (i.e., rules score vector). For the permutation vector (rules execution order) a jMetal Permutation variable representation was adopted. PMX crossover operator and Swap mutation 
were used to manipulate the real data decision variables (i.e., rules score vector). The crossover probability was $p c=0.9$ and the mutation probability was $p m=1 / n$, being $n$ the number of available filtering rules.

Table 3 shows a comparison between the results achieved using throughput heuristics (modifying only the execution scheduling) and a selection of the solutions generated by NSGA-II algorithm (optimizing both classification quality and throughput). Considering NSGA-II solutions as points in a 3D space in the form ( $F N$, FP, ExecutionTime), we included in the table the solution belonging to the pareto front closest to axis origin $(0,0,0)$ together with the best ones in each dimension (FN, FP and ExecutionTime). In the former ones, if the evaluation for a certain objective is the same, we selected the one closest to axis origin.

Table 3. Comparative benchmark results of different optimization strategies

\begin{tabular}{|c|c|c|c|}
\hline $\begin{array}{c}\text { Measure } \\
\text { Algorithm }\end{array}$ & $F N$ & $F P$ & Execution Time (ms) \\
\hline Unoptimized & \multirow{9}{*}{543} & \multirow{9}{*}{0} & 4315,846 \\
\hline GreaterABSValue & & & 3949,254 \\
\hline GreaterDistanceValue & & & 5920,114 \\
\hline IntelligentBalance & & & 5920,114 \\
\hline NegativeFirst & & & 5920,114 \\
\hline PluginGreaterSignificance & & & 5920,114 \\
\hline PluginOverloadSeparation & & & 3443,162 \\
\hline PluginSeparation & & & 3443,162 \\
\hline PositiveFirst & & & 3443,162 \\
\hline NSGA-II closest to origin & 487 & 7 & 425,036 \\
\hline NSGA-II best on FP & 543 & 0 & 435,66 \\
\hline NSGA-II best on FN & 459 & 22 & 1971,072 \\
\hline NSGA-II best on ExecutionTime & 536 & 3 & 387,136 \\
\hline
\end{tabular}

From the results included in Table 3, we can highlight the quality of the solutions generated by NSGA-II. All solutions achieved by NSGA-II outperform the ExecutionTime achieved by simple heuristics. Moreover, this algorithm also takes into consideration the minimization of errors.

In order to analyze in detail the solution space covered by NSGA-II we plotted a 3D-Pareto Front by representing all found non-dominated solutions (optimal solutions 
such no objective can be improved without sacrificing at least another one). The solutions were plotted following the equation 2

$$
\left(\frac{F N}{\text { nspam }}, \frac{F P}{\text { nham }}, \text { executionTime }\right)
$$

where nspam and nham stands for the number of spam and ham messages considered in the compilation and $F N, F P$ and executionTime are the target objectives.

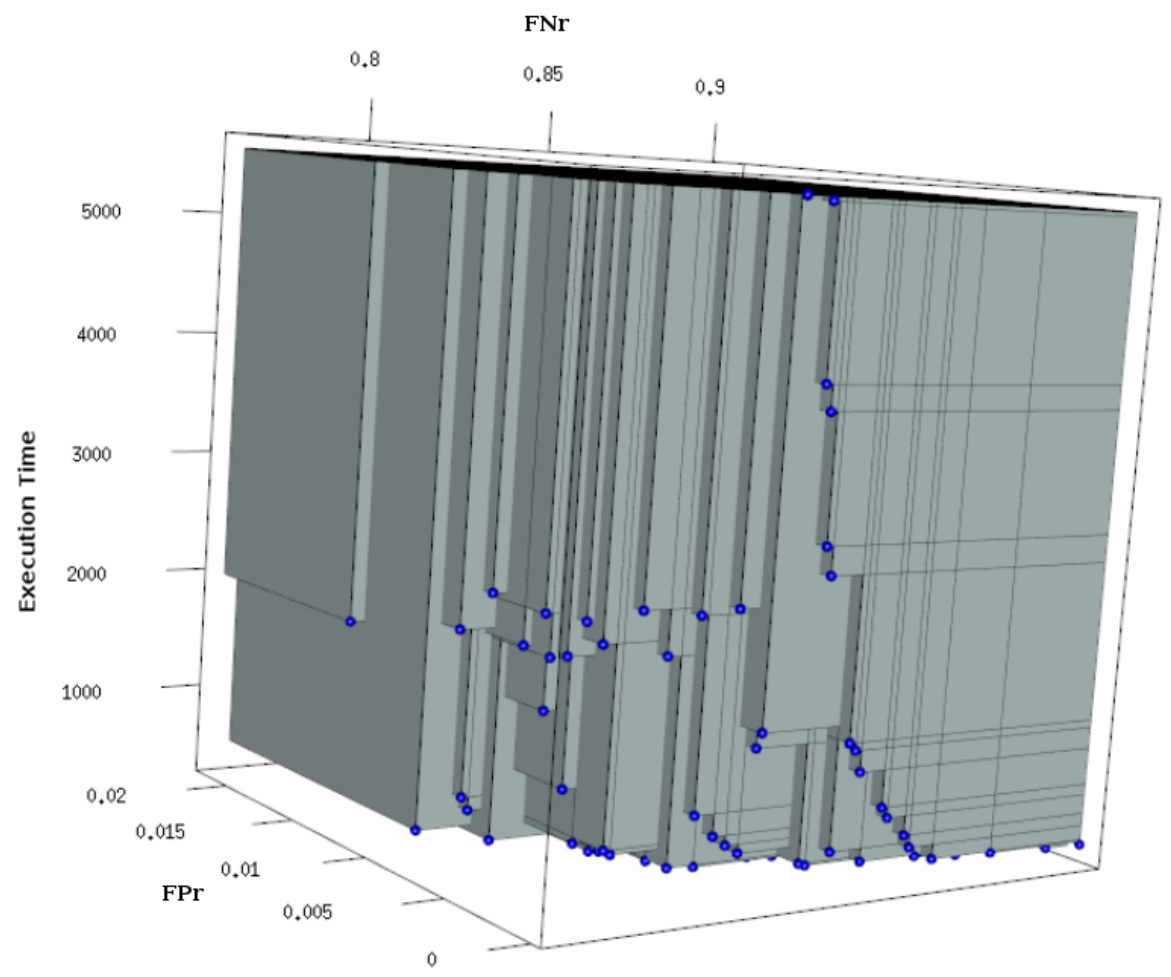

Fig. 1. 3D Pareto Front for NSGA-II execution

Figure 1 shows the hypervolume (HV) of the 3D pareto front with reference point calculated as the maximum of each dimension of the solutions set. As shown in Figure 1, the Pareto front achieved is an approximated set to the optimal by a finite number of points. The HV shown in grey in Figure 1, corresponds to a popular EMOA performance indicator, i.e., a bounded size set of points that jointly dominates a maximal part of the objective space relative to a reference point (calculated as the maximum of each dimension of the solutions set).

$\mathrm{HV}$ mean achieved in the experiments was 0,706 in comparison to 1 which represents the absolute optimum, which is a non-existent theoretical optimum. 


\section{$5 \quad$ Results discussion}

The optimization problem addressed in this study belongs to the class of optimization problems that is not possible to know the absolute optimum, therefore the results of our study have to be compared with the optimization outcomes of other state of the art algorithms applied in the anti-spam filtering domain.

One of the main advantages of EMO is its ability to provide insights of the conflicts/trade-offs among objectives of a multi-objective optimization problem.

Figure 1 highlights the tradeoff between classification time and quality. It shows that reducing the classification time is possible without significant loss of accuracy up to $1000 \mathrm{~ms}$, and reducing classification time below $1000 \mathrm{~ms}$ has a considerable impact in quality, specially expressed by the growth of FN classifications.

We can also observe that NSGA-II provides a variety of solutions for the decision maker to select according to his/her trade-offs of interest. The solutions are distributed in the solution space in a way that allows the decision maker to have a wide range of alternatives. This reveals a good performance of NSGA-II addressing this problem, i.e, it presents the best quality in comparison to other 9 heuristics of reference in the literature (Table 3), and additionally reveals a fairly good ability of generating a variety of solutions of potential interest for the decision maker.

\section{Conclusions and future work}

In this work, we have evaluated the application of evolutionary multi-objective optimization algorithms to optimize rule-based anti-spam filters classification quality and classification time (in other words, anti-spam filters effectiveness and efficiency). To this end, we presented an experimental study using RuleSIM anti-spam filtering simulator and the NSGA-II multi-objective optimization algorithm, which allows us to demonstrate the advantages of EMOA approach in setting rules relevance (scores) to minimize FP and FN spam classifications, and setting rules execution orders that allow fast classification and/or optimal tradeoffs between classification errors rates and messages classification time.

When compared to 9 state of the art heuristics for rules scheduling optimization, NSGA-II revealed the best results in all criteria. Moreover, the EMOA approach provided solutions for anti-spam filter alternative optimal settings, covering a variety of scenarios of use for the anti-spam filter. In future studies it is our intention to perform benchmarks with email corpus with other properties (in terms of size and content/domain), as well as to compare the performance of EMOA of different type, such as indicator-base and decomposition-based EMOA.

\section{Acknowledgements}

SING group thanks CITI (Centro de Investigación, Transferencia e Innovación) from University of Vigo for hosting its IT infrastructure. 
Funding: This work was partially funded by Consellería de Cultura, Educación e Ordenación Universitaria (Xunta de Galicia) and FEDER (European Union). This work was partially supported by the project Platform of integration of intelligent techniques for analysis of biomedical information (TIN2013-47153-C3-3-R) from the Spanish Ministry of Economy and Competitiveness.

\section{References}

1. Statista: The statistics portal, Global spam volume as percentage of total e-mail traffic from January 2014 to September 2016, by month (2016), https://www.statista.com/statistics/420391/spam-email-traffic-share/ (accessed 14 February 2017).

2. Digital Marketing Ramblings, 73 Incredible e-mail statistics (2016), http://expandedramblings.com/index.php/email-statistics/ (accessed 14 February 2017).

3. The Apache SpamAssassin Group, The first enterprise open-source spam filter (2003), http://spamassassin.apache.org/ (accessed 14 February 2017).

4. Méndez, J.R., Reboiro-Jato, M., Díaz, Fernando., Díaz, Eduardo., Fdez-Riverola, F.: Grindstone4Spam: An optimization toolkit for boosting e-mail classification. Journal of Systems and Software, 85(12), pp: 2909-2920 (2012), doi: 10.1016/j.jss.2012.06.027.

5. Yevseyeva, I., Basto-Fernandes, V., Ruano-Ordás, D., Méndez, J.R.: Optimising anti-spam filters with evolutionary algorithms. Expert Syst. Appl. 40(10), pp: 4010-4021 (2013), doi: 10.1016/j.eswa.2013.01.008.

6. Zhao, J., Basto-Fernandes, V., Jiao, L., Yevseyeva, I., Maulana, A., Li, R., Bäck, T., Tang, K., Emmerich, Michael T. M.: Multiobjective optimization of classifiers by means of 3D convex-hull-based evolutionary algorithms. Inf. Sci. 367-368, pp: 80-104 (2016), doi: 10.1016/j.ins.2016.05.026.

7. Basto-Fernandes, V., Yevseyeva, I., Méndez, J.R., Zhao, J., Fdez-Riverola, F., Emmerich, Michael T. M.: A spam filtering multi-objective optimization study covering parsimony maximization and three-way classification. Appl. Soft Comput. 48, pp: 111-123 (2016), doi: 10.1016/j.asoc.2016.06.043.

8. Ruano-Ordás, D., Fdez-Glez, J., Fdez-Riverola, J., Méndez, J.R.: Effective scheduling strategies for boosting performance on rule-based spam filtering frameworks. Journal of Systems and Software., 86(12), pp: 3151-3161 (2013), doi: 10.1016/j.jss.2013.07.036

9. Ruano-Ordás, D., Fdez-Glez, J., Fdez-Riverola, F., Méndez, J.R.: Combining Scheduling Heuristics to Improve e-mail Filtering Throughput. In: Proceedings of the 12th International Conference on Distributed Computing and Artificial Intelligence, Salamanca, Spain, pp: 235-242 (2015).

10. Ruano-Ordás, D., Fdez-Glez, J., Fdez-Riverola, F., Méndez, J.R.: Using new scheduling heuristics based on resource consumption information for increasing throughput on rule-based spam filtering systems. Softw., Pract. Exper. 46(8), pp: 1035-1051 (2016), doi: 10.1002/spe.2343. 
11. Ruano-Ordás, D., Fdez-Glez, J., Fdez-Riverola, F., Basto-Fernandes, V., and Méndez, J. R.: RuleSIM: a toolkit for simulating the operation and improving throughput of rule-based spam filters. Softw. Pract. Exper., 46, pp: 1091-1108 (2016), doi: 10.1002/spe.2342.

12. Deb, K., Pratap, A., Agarwal, S., Meyarivan, T.: A fast and elitist multiobjective genetic algorithm: NSGA-II, IEEE Trans. Evol. Comput. 6 (2), pp: 182-197 (2002), doi: 10.1109/4235.996017.

13. IEEE Transactions on Evolutionary Computing - Popular Documents (February 2017), http://ieeexplore.ieee.org/xpl/topAccessedArticles.jsp?punumber $=4235 \&$ so rtType=popular_most_cited_by_papers (accessed 3 April 2017).

14. The Apache SpamAssassin Group, How do I get SpamAssassin to run faster?, https://wiki.apache.org/spamassassin/FasterPerformance (accessed 14 February 2017).

15. Pérez-Díaz, N., Ruano-Ordás, D., Fdez-Riverola, F., Méndez, J.R.: Wirebrush4SPAM: a novel framework for improving efficiency on spam filtering services. Softw., Pract. Exper. 43(11), pp: 1299-1318 (2013), doi: $10.1002 /$ spe. 2135

16. The Apache SpamAssassin Group. RescoreMassCheck. Available at https://wiki.apache.org/spamassassin/RescoreMassCheck (accessed 14 February 2017).

17. Beasley, D., Possible applications of evolutionary computation, In: Evolutionary Computation 1: Basic Algorithms and Operators, 1st edition, Institute of Physics Publishing, Bristol and Philadelphia, pp. 4-18 (2000).

18. Resnick, P., RFC2822: Internet Message Format, Network Working Group, https://www.ietf.org/rfc/rfc2822.txt (accessed 14 February 2017)

19. The Apache SpamAssassin Group, The Apache SpamAssassin Public Corpus, https://spamassassin.apache.org/publiccorpus/ (accessed 14 February 2017).

20. CSMINING Group, Spam Emails Datasets, http://csmining.org/index.php/spamemail-datasets-.html (accessed 14 February 2017).

21. TREC Spam. Text REtrieval Conference. http://trec.nist.gov/data/spam.html (accessed 14 February 2017).

22. Guenter, B.: SPAM archive, http://untroubled.org/spam/ (accessed 14 February 2017).

23. Ruano-Ordás, D., Fdez-Glez, J., Fdez-Riverola, F., Basto Fernandes, V., Méndez, J.R.: RuleSIM: a toolkit for simulating the operation and improving throughput of rule-based spam filters. Softw. Pract. Exper., 46, pp: 1091-1108. (2016), doi: $10.1002 /$ spe. 2342 .

24. Durillo, J.J., Nebro, A.J.: jMetal: a java framework for multi-objective optimization, Adv. Eng. Software 42(10), pp: 760-771 (2011). doi: 10.1016/j.advengsoft.2011.05.014 